\title{
Longitudinal impedance of two-layer tube
}

\author{
M. Ivanyan and V. Tsakanov \\ Center for the Advancement of Natural Discoveries using Light Emission (CANDLE), Acharyan 31, 375040, Yerevan, Armenia
} (Received 11 August 2004; published 30 November 2004)

\begin{abstract}
The exact analytical expression for the longitudinal impedance of two-layer cylindrical tube with finite wall thickness is obtained. The numerical results for the copper-NEG, stainless steel-copper, and ceramic-copper laminated vacuum chamber impedances are given.
\end{abstract}

DOI: 10.1103/PhysRevSTAB.7.114402

PACS numbers: $41.60 .-\mathrm{m}$

\section{INTRODUCTION}

The knowledge of the vacuum chamber impedance in accelerators is an important issue to provide the stable operation of the facility from the machine performance and beam physics point of view. To adjust the technical (high vacuum performance, reduction of static charge, etc.) and beam physics (resistive instability) issues, the laminated walls of vacuum chamber parts are often used in accelerators.

The analytical solutions for impedance are known for a number of structures: two parallel plates [1], elliptic [2] and circular [3] single layer cylinders. The asymptotic and numerical study of the impedance for multilayer tube is given in a number of references [3-12]. In [13] the longitudinal impedance for two-layer tube with perfectly conducting outer layer is given.

For the multilayer pipe, the common approach to evaluate the impedance is the field matching technique. The corresponding general numerical algorithm for a straight, uniform beam in a concentric, circularcylindrical multilayer tube is derived in [4-6].

In this paper, the explicit analytical solution for longitudinal impedance of two-layer tube is obtained. The solution in ultrarelativistic limit is independent of the beam radius and therefore is valid for the pointlike charge. Based on the solution, the impedances for copperNEG (nonevaporated getter), stainless steel-copper, and ceramic-copper two-layer tubes are evaluated.

\section{ALGORITHM AND SOLUTION}

Consider the disklike plain charge $q$ (disk radius $a_{1}$ ) moving with velocity $\nu$ along the uniform, circularcylindrical two-layer tube of inner radius $a_{2}$ (Fig. 1). The disk center coincides with the tube axis. The boundary between two layers is located at $r=a_{3}$ and the outer radius of the tube is $a_{4}$. Outside of the tube is vacuum. The cross section of the tube is then divided into the five concentric regions: (1) $0 \leq r \leq a_{1}$ (vacuum), (2) $a_{1} \leq r \leq a_{2}$ (vacuum), (3) $a_{2} \leq r \leq a_{3}$ (first layer), (4) $a_{3} \leq r \leq a_{4}$ (second layer), and (5) $r \geq a_{4}$ (vacuum). The frequency domain wave equation for longitudinal electrical component $E_{z}$ in each region can be written as

$$
\frac{1}{r} \frac{d}{d r}\left(r \frac{d E_{z}^{i}}{d r}\right)-\chi_{i}^{2} E_{z}^{i}=-\frac{j \chi_{i}^{2}}{k \varepsilon_{i}} \rho_{i}, \quad(1=1,2, \ldots, 5),
$$

where $\rho_{1}=q / c \pi a_{1}^{2} e^{-j k z}$ is a charge density, $\rho_{i>1}=0, \varepsilon_{i}$ are the dielectric permeability, $\chi_{i}$ are the radial propagation constants, $k=\omega / \nu, \omega$ is the frequency. In vacuum regions $(i=1,2,5), \varepsilon_{i}=\varepsilon_{0}$ and $\chi_{i}=k / \gamma=\lambda$ with $\varepsilon_{0}$ the vacuum dielectric constant and $\gamma$ the Lorenz factor.

The general solution of the wave equation is the superposition of the particular solution and the solutions of the homogeneous one with free constants, which should be chosen to satisfy the boundary conditions. The right-hand side (rhs) of the Eq. (1) vanishes everywhere except in the beam region with nonzero charge density $\rho$ and nonzero particular solution exists only in beam region. For a uniform charge density, it is simply $E_{z}=G_{1}=j \rho / k \varepsilon_{0}$, independent of $r$. The solution of the homogeneous wave equation in the beam region, which includes the axis $r=$ 0 , is only modified Bessel functions of the first kind since those of the second kind diverge for argument zero. The longitudinal electric field in the beam region is then
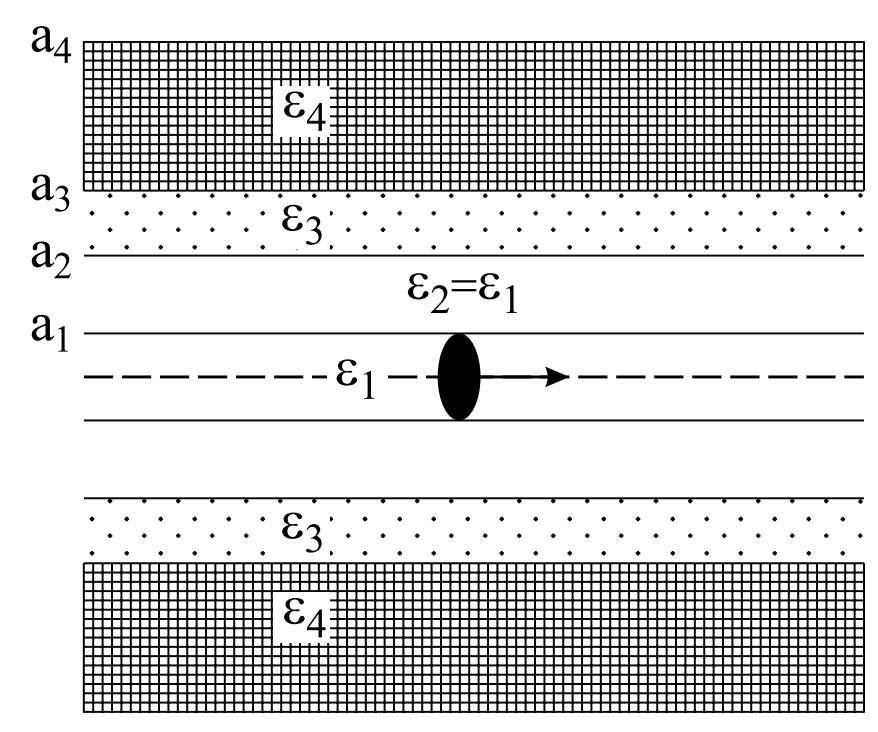

FIG. 1. Geometry of the problem. 


$$
E_{z}^{(1)}(r)=F_{1} I_{0}(\lambda r)+G_{1}
$$

In the subsequent regions $2,3,4$, the longitudinal electric field is given by superposition of modified Bessel functions of both kinds:

$$
E_{z}^{(i)}=F_{i} R_{i}(r)+G_{i} S_{i}(r), \quad i=2,3,4,
$$

with $F_{i}, G_{i}$ unknown coefficients and $R_{i}(r), S_{i}(r)$ the new functions combined as

$$
\begin{aligned}
R_{i}(r) & =K_{0}\left(\chi_{i} a_{i}\right) I_{0}\left(\chi_{i} r\right)-I_{0}\left(\chi_{i} a_{i}\right) K_{0}\left(\chi_{i} r\right), \\
S_{i}(r) & =-K_{1}\left(\chi_{i} a_{i}\right) I_{0}\left(\chi_{i} r\right)-I_{1}\left(\chi_{i} a_{i}\right) K_{0}\left(\chi_{i} r\right) .
\end{aligned}
$$

In the outer region $(i=5)$ that extends to infinity, only modified Bessel functions of the second kind are admissible, as those of the first kind diverge at infinity. The electric field in the outer region is then

$$
E_{z}=F_{5} K_{0}(\lambda r) \text {. }
$$

The unknown coefficients $F_{i}(i=1, \ldots, 5)$ and $G_{i}(i=$ $2,3,4)$ are defined by the matching conditions at transition boundaries:

$$
\left\{\begin{array}{l}
E_{z}^{(i)}\left(\chi_{i} a_{i}\right)=E_{z}^{(i+1)}\left(\chi_{i} a_{i}\right), \\
H_{\theta}^{(i)}\left(\chi_{i} a_{i}\right)=H_{\theta}^{(i+1)}\left(\chi_{i} a_{i}\right),
\end{array} \quad i=1,2,3,4\right.
$$

where tangential magnetic field component $H_{\theta}^{(i)}$ is defined by tangential electrical field component as

$$
H_{\theta}^{(i)}(r)=\frac{j \omega \varepsilon_{i}}{\chi_{i}^{2}} \frac{\partial E_{z}^{(i)}(r)}{\partial r}
$$

Matching conditions (6) consist of four pairs of equations. This system of equations can be solved consecutively: the first pair of equations that contains unknown parameter $F_{1}$ is solved with respect to $F_{2}$ and $G_{2}$. Substituting these coefficients into the next pair of equations, the coefficients $F_{3}$ and $G_{3}$ are obtained as linear parametric expressions with respect to parameters $F_{1}$ and so on. The last pair of equations is then the system of linear equations with respect to unknown parameters $F_{1}$ and $F_{5}$. The solution for $F_{1}$ is then given by

$$
F_{1}=G_{1} a_{1} \chi_{1} \frac{\sum_{i=1}^{8} \beta_{i} \alpha_{i} f_{i}}{\sum_{i=1}^{8} \gamma_{i} \alpha_{i} f_{i}}
$$

where

$$
\begin{aligned}
& \alpha_{1}=\varepsilon_{1} \varepsilon_{3}^{2} \chi_{1} \chi_{4}^{2}, \quad \alpha_{2}=\varepsilon_{1}^{2} \varepsilon_{3} \chi_{3} \chi_{4}^{2}, \\
& \alpha_{3}=\varepsilon_{1}^{2} \varepsilon_{4} \chi_{3}^{2} \chi_{4}, \quad \alpha_{4}=\varepsilon_{1} \varepsilon_{4}^{2} \chi_{1} \chi_{3}^{2}, \\
& \alpha_{5}=\varepsilon_{3} \varepsilon_{4}^{2} \chi_{1}^{2} \chi_{3}, \quad \alpha_{6}=\varepsilon_{3}^{2} \varepsilon_{4} \chi_{1}^{2} \chi_{4}, \\
& \alpha_{7}=\alpha_{8}=\varepsilon_{1} \varepsilon_{3} \varepsilon_{4} \chi_{1} \chi_{3} \chi_{4},
\end{aligned}
$$

and

$$
\begin{aligned}
& \beta_{1}=S_{1}\left(a_{2}\right), \quad \gamma_{1}=I_{0}\left(a_{2} \chi_{1}\right), \quad f_{1}=-K_{1}\left(a_{4} \chi_{1}\right) S_{3}^{\prime}\left(a_{2}\right) R_{4}\left(a_{3}\right), \quad \beta_{2}=S_{1}^{\prime}\left(a_{2}\right), \quad \gamma_{2}=I_{1}\left(a_{2} \chi_{1}\right), \\
& f_{2}=K_{1}\left(a_{4} \chi_{1}\right) S_{3}\left(a_{2}\right) R_{4}\left(a_{3}\right), \quad \beta_{3}=S_{1}^{\prime}\left(a_{2}\right), \quad \gamma_{3}=I_{1}\left(a_{2} \chi_{1}\right), \quad f_{3}=-K_{1}\left(a_{4} \chi_{1}\right) R_{3}^{\prime}\left(a_{2}\right) R_{4}^{\prime}\left(a_{3}\right), \\
& \beta_{4}=S_{1}^{\prime}\left(a_{2}\right), \quad \gamma_{4}=I_{1}\left(a_{2} \chi_{1}\right), \quad f_{4}=-K_{0}\left(a_{4} \chi_{1}\right) R_{3}\left(a_{2}\right) S_{4}^{\prime}\left(a_{3}\right), \quad \beta_{5}=S_{1}\left(a_{2}\right), \quad \gamma_{5}=I_{0}\left(a_{2} \chi_{1}\right), \\
& f_{5}=K_{0}\left(a_{4} \chi_{1}\right) R_{3}\left(a_{2}\right) S_{4}^{\prime}\left(a_{3}\right), \quad \beta_{6}=S_{1}\left(a_{2}\right), \quad \gamma_{6}=I_{0}\left(a_{2} \chi_{1}\right), \quad f_{6}=-K_{0}\left(a_{4} \chi_{1}\right) S_{3}^{\prime}\left(a_{2}\right) S_{4}\left(a_{3}\right), \\
& \beta_{7}=-R_{1}^{\prime}\left(a_{2}\right), \quad \gamma_{7}=I_{0}\left(a_{2} \chi_{1}\right), \quad f_{7}=K_{1}\left(a_{4} \chi_{1}\right) R_{3}^{\prime}\left(a_{2}\right) R_{4}^{\prime}\left(a_{3}\right), \quad \beta_{8}=-S_{1}^{\prime}\left(a_{2}\right), \quad \gamma_{8}=I_{1}\left(a_{2} \chi_{1}\right), \\
& f_{8}=K_{0}\left(a_{4} \chi_{1}\right) S_{3}\left(a_{2}\right) S_{4}\left(a_{3}\right) \text {. }
\end{aligned}
$$

In the expressions above

$$
\begin{aligned}
& R_{i}^{\prime}(r)=K_{0}\left(\chi_{i} a_{i}\right) I_{1}\left(\chi_{i} r\right)+I_{0}\left(\chi_{i} a_{i}\right) K_{1}\left(\chi_{i} r\right), \\
& S_{i}^{\prime}(r)=-K_{1}\left(\chi_{i} a_{i}\right) I_{1}\left(\chi_{i} r\right)+I_{1}\left(\chi_{i} a_{i}\right) K_{1}\left(\chi_{i} r\right) .
\end{aligned}
$$

Further, we consider the ultrarelativistic case $(\gamma \rightarrow \infty)$. The formula (8) in the ultrarelativistic case is simplified to

$$
F_{1}(\nu=c)=-G_{1}+G_{1} a_{1}^{2} U^{-1},
$$

with

$$
\begin{aligned}
U= & a_{2}^{2}-2 \frac{a_{2} \varepsilon_{3}}{\chi_{3} \varepsilon_{0}} \\
& \times \frac{\varepsilon_{3} \chi_{4} R_{4}\left(a_{3}\right) S_{3}^{\prime}\left(a_{2}\right)-\varepsilon_{4} \chi_{3} R_{3}^{\prime}\left(a_{2}\right) R_{4}^{\prime}\left(a_{3}\right)}{\varepsilon_{3} \chi_{4} R_{4}\left(a_{3}\right) S_{3}\left(a_{2}\right)-\varepsilon_{4} \chi_{3} R_{3}\left(a_{2}\right) R_{4}^{\prime}\left(a_{3}\right)} .
\end{aligned}
$$

The longitudinal electrical field component in the beam region $0 \leq r \leq a_{1}$ is then given by

$$
E_{z}^{(1)}(r)=G_{1} a_{1}^{2} U^{-1}=\frac{j q}{c \pi k \varepsilon_{0} U} e^{-j k z} .
$$

Expression (14) is independent of beam radius $a_{1}$ and therefore is valid for the pointlike charge. The monopole longitudinal impedance is then defined by

$$
Z(k)=-\frac{j Z_{0}}{\pi k U},
$$

with $Z_{0}=377 \Omega$ impedance of free space.

\section{SPECIAL CASES}

In this section some special cases that follow from the general expressions (13) and (15) are presented.

Single layer tube.-For the single layer cylindrical tube with finite wall thickness $\left(a_{2}=a_{3}=a\right)$, the general expression for $U$ is modified to 


$$
U=a^{2}-2 \frac{a \varepsilon}{\varepsilon_{0} \chi} \frac{K_{0}(\chi b) I_{1}(\chi a)+I_{0}(\chi b) K_{1}(\chi a)}{K_{0}(\chi b) I_{0}(\chi a)-I_{0}(\chi b) K_{0}(\chi a)},
$$

where $\varepsilon$ and $\chi$ are the dielectric permeability and radial propagation constant of the tube wall, respectively, $a$ and $b$ are the inner and outer radii of the tube.

Infinite wall thickness.-For infinite wall thickness $(b \rightarrow \infty)$, formula (16) coincides with the well-known expression for the impedance derived by Chao [3]

$$
U=a^{2}-2 a \frac{\varepsilon \nu H_{1}^{(1)}(\nu a)}{k^{2}\left(\varepsilon-\varepsilon_{0}\right) H_{0}^{(1)}(\nu a)},
$$

where $\nu=j \chi$ and $H_{i}^{(1)}(\nu a)$ are the first kind Hankel functions.

Metallic-type layers.-For the metallic-type layers, the dielectric permeability $\varepsilon_{i}$ and the radial propagation constants $\chi_{i}(i=3,4)$ are given by

$$
\varepsilon_{i}=\varepsilon_{0}-j \sigma_{i} / \omega, \quad \chi_{i}=\sqrt{j \mu_{0} \bar{\sigma}_{i} \omega}
$$

where $\sigma_{i}$ are the layers' conductivities, $\mu_{0}$ is the vacuum magnetic permeability, and the positive real part of squared root is assumed. A good approximation for metallic-type two-layer tube is the smallness of each layer skin depth $\delta_{i}=\left(2 / \sigma_{i} \mu_{0} \omega\right)^{1 / 2}$ with respect to the layer inner radius $a_{i-1}$, i.e., $\delta_{i} / a_{i-1} \ll 1(i=3$, 4). For two-layer metallic tube, formula (13) is then simplified to

$$
U=a^{2}+2 \frac{a \varepsilon_{3}}{\varepsilon_{0} \chi_{3}} \frac{1+\alpha \operatorname{th}\left(\chi_{3} d_{3}\right) \operatorname{th}\left(\chi_{4} d_{4}\right)}{\operatorname{th}\left(\chi_{3} d_{3}\right)+\alpha \operatorname{th}\left(\chi_{4} d_{4}\right)},
$$

where $\alpha=\frac{\chi_{4}}{\chi_{3}} \frac{\varepsilon_{3}}{\varepsilon_{4}} \approx\left(\sigma_{3} / \sigma_{4}\right)^{1 / 2}, a=a_{2}$ is the inner radius of the tube, $d_{3}, d_{4}$ are the layers' thickness. For the single layer tube $(\alpha=1)$ with wall thickness $d$ and dielectric permeability $\varepsilon$, formula (19) gives

$$
U=a^{2}+2 \frac{a \varepsilon}{\varepsilon_{0} \chi} c t h \chi d
$$

that for $d \rightarrow \infty$ coincides with truncated impedance of the infinite thickness tube [3]. For perfectly conducting outer layer $\sigma_{4}=\infty(\alpha=0)$, the solution coincides with impedance given in [13]. Note that actually for all the practical applications, the formula (19) is valid and well approximates the exact solution given by (13).

Ceramic pipe.-An important case is the ceramic pipe with metallic coating. The permeability of ceramic is given by $\varepsilon=\varepsilon_{0} n^{2}$ with $n$ the refraction index. The radial propagation constant in ceramic material without losses is the imaginary quantity and equal to $\chi=j k \sqrt{n^{2}-1}$. For practical application, the good approximation of the impedance follows from $\left|a_{2} \chi_{3}\right| \gg 1$ (in metallic layer) and $\left|a_{3} \chi_{4}\right|=k a_{3} \sqrt{n^{2}-1} \gg 1$ (in ceramic layer). The impedance (13) is then modified to

$$
U=a^{2}+2 \frac{a \varepsilon_{3}}{\varepsilon_{0} \chi_{3}} \frac{1-j \alpha \operatorname{th}\left(\chi_{3} d_{3}\right) \operatorname{tg}\left(k n^{\prime} d_{4}\right)}{\operatorname{th}\left(\chi_{3} d_{3}\right)-j \alpha \operatorname{tg}\left(k n^{\prime} d_{4}\right)},
$$

where $n^{\prime}=\sqrt{n^{2}-1}$. As it follows from (21), the impedance of metallic covered ceramic pipe is an oscillating function. At frequencies $k n^{\prime} d_{4}=\pi l(l=0,1,2,3)$, the impedance is given by finite thickness metallic tube impedance (20). At frequencies $k n^{\prime} d_{4}=\pi / 2+\pi l(l=$ $0,1,2,3)$, the impedance is given by

$$
U=a^{2}+2 \frac{a \varepsilon_{3}}{\varepsilon_{0} \chi_{3}} \operatorname{th}\left(\chi_{3} d_{3}\right) .
$$

For both sets of frequencies, the impedance is independent of the ceramic layer.

\section{LOW AND HIGH FREQUENCY ASYMPTOTES FOR METALLIC TUBE}

The low frequency asymptote of the two-layer metallic tube impedance is given by the lowest term in the expansion of the exact impedance (13) into the series with respect to $\omega / \omega_{a i}$, where $\omega_{a i}=1 / \mu_{0} \sigma_{i} a_{i-1}^{2}(i=3,4)$ :

$$
\begin{aligned}
Z(k)= & -\frac{Z_{0}}{2 \pi}\left\{j k \ln \left(\frac{a_{2}}{a_{4}}\right)+\frac{k^{2}}{2} Z_{0}\left[a_{2}^{2} \sigma_{3} L_{2}-\frac{1}{2} a_{4}^{2} \sigma_{4}\right.\right. \\
& \left.\left.+a_{3}^{2}\left(\sigma_{4}-\sigma_{3}\right) L_{3}\right]\right\},
\end{aligned}
$$

with

$$
\begin{aligned}
L_{2} & =\frac{1}{2}+\ln \frac{a_{2}}{a_{4}}\left(\ln \frac{a_{2}}{a_{4}}-1\right), \\
L_{3} & =\frac{1}{2}+\ln \frac{a_{3}}{a_{4}}\left(\ln \frac{a_{3}}{a_{4}}-1\right) .
\end{aligned}
$$

The asymptote (23) is valid for the frequency range of $\omega / \omega_{A} \ll 1\left[\omega_{A}=\min \left(\omega_{a 3}, \omega_{a 4}\right)\right]$ when for both layers the skin depth $\delta_{i}$ is larger than the inner radius $a_{i-1}$, i.e., $\delta_{i} / a_{i-1} \gg 1$. Note that the imaginary (reactive) term of the asymptote is independent of the layer's material.

However, for the most of practical applications the contribution of the very low frequency part to the overall impedance is negligibly small. The impedance is basically dominated by the spectral part of electromagnetic fields, where the layers' skin depths $\delta_{i}$ are smaller with respect to the layer inner radius $a_{i-1}$, i.e., $\delta_{i} / a_{i-1} \ll 1$ and the presentation of general impedance (13) by truncated impedance (19) is valid. In high frequency asymptotic presentation of the impedance (13), the skin depth of the inner layer $\delta_{3}$ is much smaller than the layer thickness $d_{3}$, i.e., $\delta_{3} / d_{3} \ll 1$, and the asymptote is defined by the inner layer contribution

$$
Z(k)=-\frac{j Z_{0}}{\pi k}\left(a^{2}+2 \frac{a \varepsilon_{3}}{\varepsilon_{0} \chi_{3}}\right)^{-1},
$$

where $a$ is the inner layer radius. In terms of the dimensionless wave number $\kappa=s_{0} \omega / c$, where $s_{0}=$ $\left(2 c a^{2} \varepsilon_{0} / \sigma_{3}\right)^{1 / 3}$ is the characteristic distance for the inner layer, we get 


$$
Z(k)=\frac{Z_{0} s_{0}}{2 \pi a^{2}}\left(j \frac{\kappa}{2}+\frac{(1+j)}{2} \sqrt{\kappa} \frac{s_{0}^{2}}{a^{2}}+(1-j) \frac{1}{\sqrt{\kappa}}\right)^{-1}
$$

that for $s_{0}^{2} / a^{2} \ll 1$ coincides with the well-known truncated impedance presentation for the single layer tube of the radius $a$ and infinite thickness given by Chao [3]. By keeping higher order terms of $\kappa$ in the real and imaginary parts of (26), the asymptotic presentation of the impedance is simplified to

$$
Z(\omega)=-\frac{Z_{0}}{\pi a^{2} k}\left(\frac{1}{a k} \sqrt{\frac{2 Z_{0} \sigma_{3}}{k}}-j\right) .
$$

Note that the impedance presentation (25) is valid for the frequency range of $\omega / \omega_{D} \gg 1\left[\omega_{D}=\right.$ $\left.\max \left(\omega_{a 3}, \omega_{a 4}, \omega_{d 3}\right) ; \omega_{d 3}=1 / \mu_{0} \sigma_{3} d_{3}^{2}\right]$, and for the thick inner layer $\left(d_{3} \sim a\right)$ the low frequency approximation for the truncated impedance (19) can be given by

$$
Z(\omega)=-\frac{Z_{0}}{2 \pi a}(1+j) \sqrt{\frac{k}{2 Z_{0} \sigma_{3}}}
$$

that coincides with low frequency approximation of the truncated impedance for the single layer tube with infinite thickness [3].

For the thin layers $d_{i} \ll \delta_{i}$, when the skin depth is larger than the layer's thickness, the truncated impedance (19) in the frequency range $\max \left(\omega_{a 3}, \omega_{a 4}\right) \ll \omega \ll$ $\min \left(\omega_{d 3}, \omega_{d 4}\right)$ is approximated by

$$
Z(k)=-\frac{j Z_{0} \varepsilon_{0} \chi_{3}}{\pi k a\left[a \varepsilon_{0} \chi_{3}+6 \varepsilon_{3}\left(3 \chi_{3}\left(d_{3}+d_{4}\right)-\chi_{3}^{3} d_{3}^{3}-\alpha \chi_{4}^{3} d_{4}^{3}\right)^{-1}\right]},
$$

which actually gives the low frequency approximation of the truncated impedance (13) in this particular case:

$$
Z(k)=\frac{Z_{0}}{2 \pi a}\left[j\left(d_{3}+d_{4}\right) k+\frac{1}{3} Z_{0} k^{2}\left(\sigma_{3} d_{3}^{3}+\sigma_{4} d_{4}^{3}\right)\right] .
$$

Finally, for the special case of very thin inner layer $d_{3} / \delta_{3} \ll \min \left(\alpha, \alpha^{-1}\right)$ and the thick outer layer $d_{4} \geq$ $a_{3} \gg \alpha d_{3}$, the low frequency part of the truncated impedance (19) $\left[\max \left(\omega_{a 3}, \omega_{a 4}\right) \ll \omega \ll \omega_{d 3}\right]$ is given by the outer layer

$$
Z(k)=-\frac{j Z_{0}}{\pi k}\left(a^{2}+2 \frac{a \varepsilon_{4}}{\varepsilon_{0} \chi_{4}}\right)^{-1} .
$$

Further simplification of expression (31) gives the usual low frequency approximation [3]:

$$
Z(\omega)=-\frac{Z_{0}}{2 \pi a}(1+j) \sqrt{\frac{k}{2 Z_{0} \sigma_{4}}}
$$

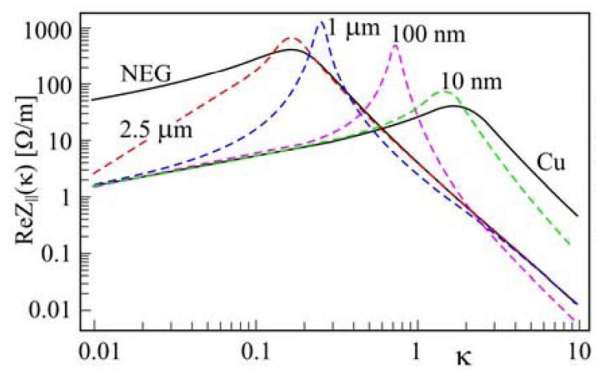

\section{NUMERICAL EXAMPLES}

In this section, the longitudinal impedance evaluation for copper-NEG, stainless steel-copper, and ceramiccopper two-layer tubes is presented. The first material is the frame tube while the second is the cover. The materials conductivities $\sigma$ are taken: NEG: $5.5 \times$ $10^{4} \Omega^{-1} \mathrm{~m}^{-1}$; stainless steel: $0.14 \times 10^{7} \Omega^{-1} \mathrm{~m}$; copper: $5.88 \times 10^{7} \Omega^{-1} \mathrm{~m}^{-1}$. The inner and outer radii of the tube are $a=5 \mathrm{~mm}$ and $b=7 \mathrm{~mm}$, respectively.

Figure 2 presents the longitudinal impedances of the copper-NEG (left) and stainless steel-copper (right) tubes for the various thickness of cover material: (NEG: 2.5, 1, 0.1, $0.01 \mu \mathrm{m}$; Cu: 1, 10, $100 \mathrm{~nm}$ ). The horizontal axis is the dimensionless wave number $\kappa=k s_{0}$ with $s_{0}=$ $\left(2 c a^{2} \varepsilon_{0} / \sigma\right)^{1 / 3}$ the characteristic distance of the copper tube with radius $a$ and conductivity $\sigma$. The solid curves show the impedances of conventional homogeneous tubes (copper, NEG, stainless steel) with infinite thickness and inner radius of $a$. In the frequency region, where the skin depth is larger than cover thickness, the impedance of the frame tube basically contributes to the two-layer tube impedance. At the frequency region, where the skin depth

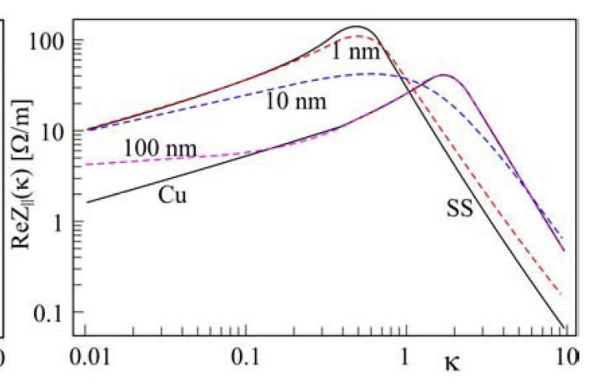

FIG. 2. (Color) Real part of copper-NEG (left) and stainless steel-copper (right) tube impedances for various cover thickness. 

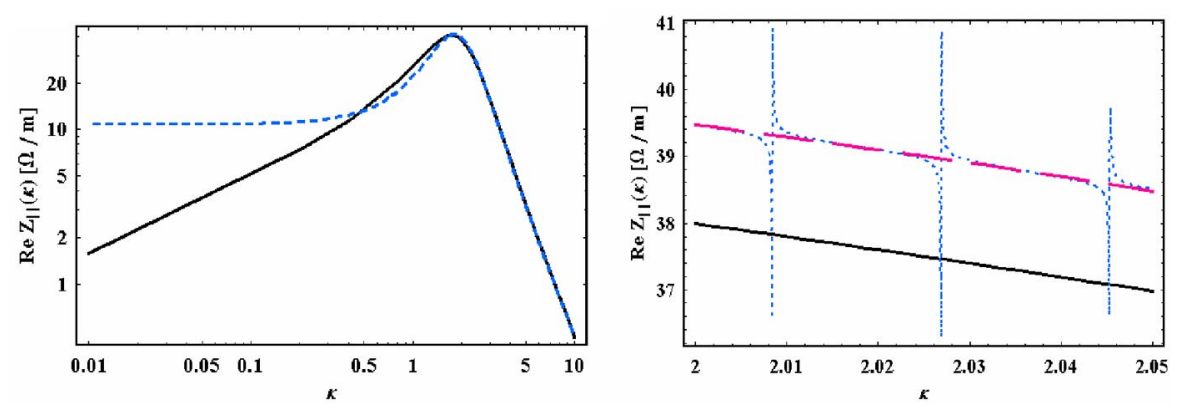

FIG. 3. (Color) Real part of the longitudinal impedance for the lossless ceramic pipe with copper cover. Left: the smooth part of the impedance (dotted curve); the vertical axis has logarithmical scale. Right: the fine structure of impedance (dotted line) and its smooth part (dashed line). The solid curve is the impedance of homogeneous tube with infinite copper wall.

is smaller than the cover thickness, the cover layer of the tube defines the impedance in agreement with (25). As a result, with increasing the cover thickness, the transition of the frame tube impedance to the cover tube impedance is first observed at the high frequency region of the impedance.

Figure 3 presents the longitudinal impedance of ceramic tube with finite wall thickness and inner metallic cover (copper). The inner and outer radii of the pipe are 5 and $7 \mathrm{~mm}$, respectively, and the cover thickness is $50 \mathrm{~nm}$, the refraction index of ceramics is $n=1.5$. The impedance is presented as a superposition of the smooth curve (Fig. 3, left) with quasiperiodical modulation (Fig. 3, right). The smooth part of the impedance is independent of lossless ceramic layer and is given by (22). The ceramic layer leads to quasiperiodical modulation of the impedance with narrow resonances at $k n^{\prime} d_{4}=\pi / 2+\pi l$ $(l=0,1,2,3, \ldots)$. The period of oscillations is inversely proportional to $n^{\prime}$.

\section{CONCLUSION}

The impedance for the two-layer tube with finite wall thickness is obtained. The result is an exact analytical solution for the ultrarelativistic case, applicable for any two-layer tube with arbitrary material and thickness. A good approximation for impedance of metallic-type two- layer tube is derived that can be useful for practical applications.

[1] H. Henke and O. Napoly, in Proceedings of the Second European Particle Accelerator Conference, Nice, France, 1990 (Editions Frontiers, Gif-sur-Yvette, 1991), pp. 1046-1048.

[2] A. Piwinski, Report No. DESY-94-068, 1994, p. 23.

[3] A.W. Chao, Technical Report No. 2946, SLAC-PUB, 1982; see also A.W. Chao, Physics of Collective Beam Instabilities in High Energy Accelerators (Wiley, New York, 1993).

[4] B. Zotter, Part. Accel. 1, 311 (1970).

[5] B. Zotter, CERN Technical Report No. 69-15, 1969.

[6] B.W. Zotter and S. A. Kheifets, Impedances and Wakes in High-Energy Particle Accelerators (World Scientific, Singapore, 1997).

[7] J. D. Jackson, SSCL Report No. SSC-N-110, 1986.

[8] D. Briggs, SSCL Report No. SSCL-512, 1992.

[9] E. Keil and B. Zotter, in Proceedings of the European Particle Accelerator Conference (EPAC'98), Stockholm, 1998 (IOP, Philadelphia, 1998), pp. 963-965.

[10] A. Piwinski, IEEE Trans. Nucl. Sci. 24, 1364 (1977).

[11] G. R. Lambertson, LBL Report No. LBL-44454, 1999.

[12] F. Ruggiero, Part. Accel. 50, 83 (1995).

[13] A. Burov and A. Novokhatskii, INP-Novosibirsk, Report No. 90-28, 1990. 